\title{
MICROMANIPULATOR CONTROLLED FABRICATION OF MICRO- AND NANOSCALE POLYMER FIBERS AND APPLICATION AS SACRIFICIAL STRUCTURES IN THE PRODUCTION OF MICROCHANNELS
}

\author{
$\underline{\text { Scott M. Berry }}^{1}$, Thomas J. Roussel ${ }^{1,2}$, Scott D. Cambron ${ }^{1,2}$, Steven A. Harfenist ${ }^{3 *}$, Robert W. Cohn ${ }^{3}$, and \\ Robert S. Keynton ${ }^{1,2,3}$ \\ Departments of Mechanical Engineering ${ }^{1}$ and Bioengineering ${ }^{2}$, and \\ the ElectroOptics Research Institute and Nanotechnology Center ${ }^{3}$ \\ University of Louisville, Louisville, KY, USA \\ * Current address for SAH is Department of Physics, California Polytechnic State University \\ San Luis Obispo, CA, USA
}

\begin{abstract}
Suspended micro- and nanoscale polymer fibers were created using a new and novel technique which is performed at room temperature. This method involves drawing solvated PMMA into liquid filaments, which thin, via surface tension, as the solvent evaporates, subsequently yielding a solid fiber. The PMMA solution was drawn by two separate techniques: 1) using a stylus to extrude a solution filament between two pre-deposited pools, and 2) using a pressurized capillary loaded with solution to directly deposit filaments of solution on the substrate in a "connect-thedots" style. Also, because both the stylus and pressurized capillary were controlled with an ultra-high precision instrument, the fibers can be precisely positioned in three dimensions. The diameters of these fibers were controlled, within the range of $450 \mathrm{~nm}$ to 100 $\mu \mathrm{m}$, by varying the molecular weight of the polymer or the concentration of the PMMA solution. It was discovered that increasing either or both of these variables led to an increase in fiber diameter. Also, the effect of stylus material on fiber diameter variance was examined, with a Parylene ${ }^{\circledR}$-coated stylus yielding the lowest overall variance. These fibers were coated with glass and Parylene ${ }^{\circledR}$ and dissolved to produce hollow microchannels suitable for electroosmotic flow. Electroosmotic channels with diameters ranging from $4 \mu \mathrm{m}$ to $100 \mu \mathrm{m}$ were successfully fabricated and tested.
\end{abstract}

\section{INTRODUCTION}

A new technique for fabricating micro- and nanoscale polymer fibers has been discovered and was reported in $[\mathbf{1}, \mathbf{2}]$. This technique involves drawing a filament of solvated polymer at room temperature between two points and allowing surface tension forces to thin the diameter of the filament to the micro- or nanoscale before evaporation of the solvent leads to the solidification of the filament into a fiber. These structures are fabricated in three dimensions, providing an escape from the constraints of conventional planar fabrication. It has been suggested by [1] that fibers created with this process could be used as microoptical couplers, sacrificial backbones for microfluidic devices, or three-dimensional (3-D) electrical connectors (via applying this method to conductive polymers).

The present study describes the characterization of this process, enabling a user to control the fiber diameter by varying the experimental parameters, such as the polymer molecular weight and solvated polymer solution concentration. Additionally, this paper demonstrates the microfluidic applicability of this fabrication method by utilizing the polymer fibers as sacrificial backbone structures in the formation of suspended microchannels. Electroosmotic flow is induced in these microchannels to further validate this procedure as a viable methodology for the construction of 3-D microchannels.

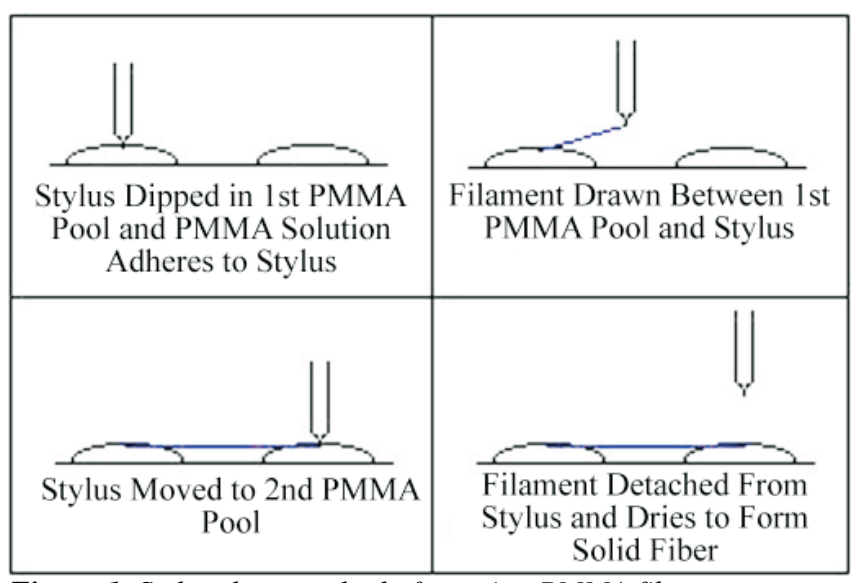

Figure 1. Stylus-draw method of creating PMMA fibers.

\section{FABRICATION}

Initially, fibers were drawn from reservoirs of solvated poly-methyl methacrylate (PMMA) (MicroChem) using a tungsten stylus (tip radius $=20 \mathrm{~nm}$ ) to create a filament of solution between the two reservoirs (Fig. 1). As the solvent (chlorobenzene) evaporates, surface tension forces cause the filament to thin, resulting in the formation of a cylindrical polymer fiber with a nearly uniform cross-section Precise positioning of the fibers was accomplished by controlling the stylus with a programmable, custom-made ultra-high-precision micromilling machine (MMM) (Dover Instruments, Inc.) [3].

An alternative, direct-write, method of fiber drawing was also developed. This new technique involved loading a glass capillary (1-mm I.D.) with solvated PMMA and utilizing it to both deposit the reservoirs and draw the fibers, eliminating the need for the low-precision, manual reservoir deposition in the method previously described (Fig. 2). Manipulation of the direct-write capillary process was also controlled with the MMM, enabling nanoscale positioning resolution.

Channels were fabricated by coating the PMMA fibers with a layer of borosilicate glass (BSG) followed by Parylene ${ }^{\circledR}$. BSG was deposited via RF sputtering (Technics 4604) to a thickness of $25 \mathrm{~nm}$ to establish a hydrophilic interior channel wall. A $10 \mu \mathrm{m}$ Parylene ${ }^{\circledR}$ layer (SCS Parylene Deposition System 2010) was included to provide structural reinforcement for the fragile BSG thin wall. $500 \mu \mathrm{m}$-diameter holes were drilled into the coated and dried PMMA reservoirs to provide access to the PMMA and completed channels. The entire platform was submerged in acetone 


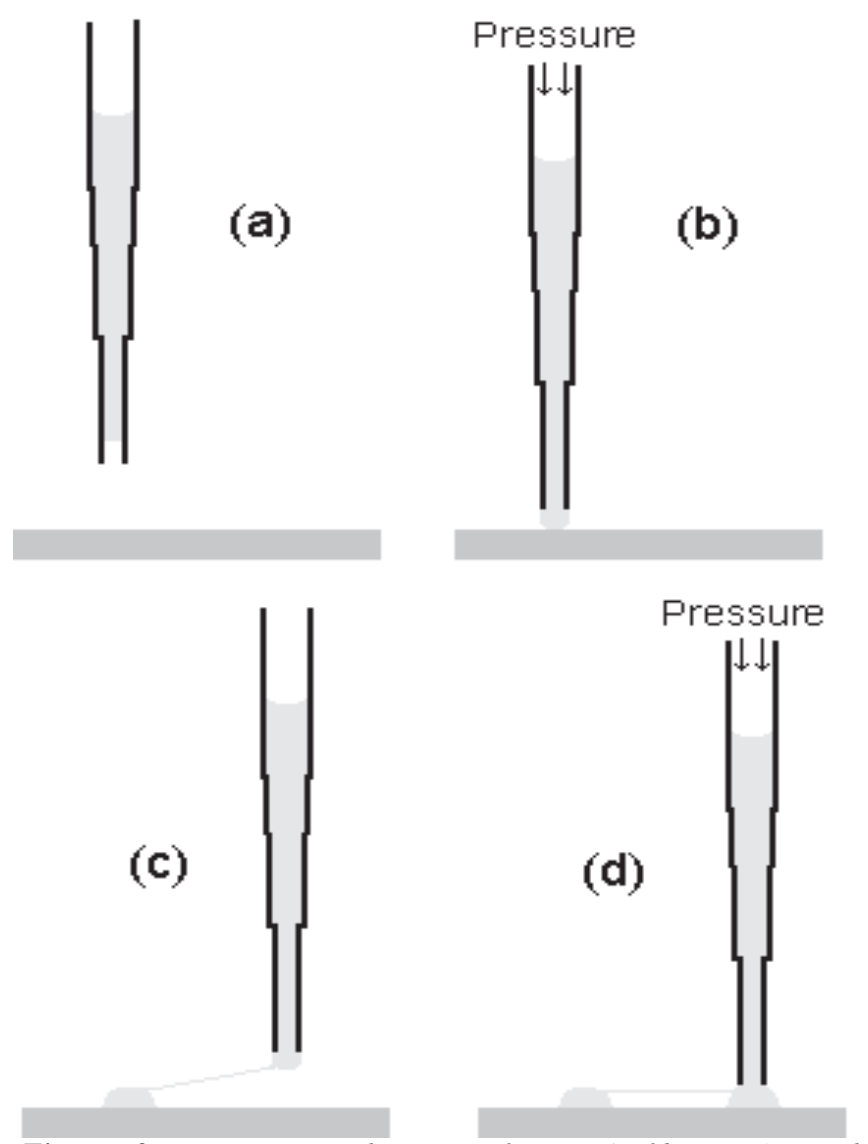

Figure 2. Direct-write drawing of PMMA fibers, a) Load capillary with solvated PMMA, b) Pressurize capillary to expel solution into contact with substrate, c) Translate capillary to desired endpoint, d) Pressurize capillary to allow PMMA solution to contact substrate.

for 7 days to ensure total dissolution of the shrouded PMMA matrix, leaving a hollow tube of BSG and Parylene ${ }^{\circledR}$. Finally, the channels were dried with liquid carbon dioxide using a Samdri Critical Point Dryer (Tousimis) (Fig. 3).

All microchannel fabrication was performed on substrates containing a pair of microfabricated planar gold electrodes separated by a $1.8 \mathrm{~mm}$ wide trench created with a dicing saw (Disco DAD321). The channels were loaded with a phosphate buffer solution (10 mM, pH 6.1) containing 1\% (by vol) of $20 \mathrm{~nm}-$ diameter fluorescent tracer particles (Molecular Probes) which were subsequently excited and imaged at $5 \mathrm{~Hz}$ using a low-light, high-resolution CCD camera (Roper Scientific) affixed to a fluorescent microscope (Olympus). The potential applied to the gold electrodes was modulated from $0 \mathrm{~V}$ to $1 \mathrm{kV}$ with the polarity being alternated between a positive and negative potential via a high voltage power supply (CZE1000PN30, Spellman High Voltage Electronics Corp.).

\section{RESULTS}

The stylus-draw method was utilized to produce fibers with diameters ranging between $450 \mathrm{~nm}$ to $100 \mu \mathrm{m}$ suspended between the two reservoirs (Fig. 4a,c). In general, these fibers have a uniform cross section that tapers slightly at the fiber / reservoir interface (Fig. 4d). Fibers were drawn from concentrations ranging from $15.5 \%$ to $21.6 \%$ (by weight) for the $495 \mathrm{k} \mathrm{g} / \mathrm{mol}$ solution and $13 \%$ to $21.3 \%$ (by weight) for the $950 \mathrm{k} \mathrm{g} / \mathrm{mol}$ solution.

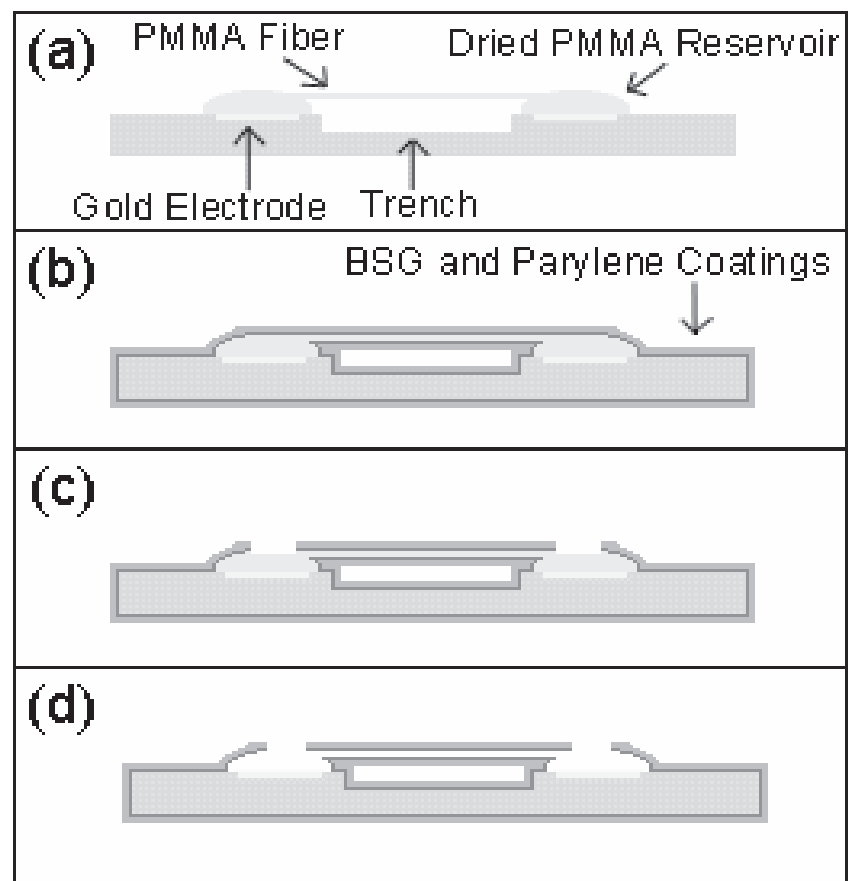

Figure 3. Fabrication of channels from PMMA fiber, a) PMMA fiber (light blue) between two PMMA reservoirs on glass substrate (gray), b) Deposition of BSG and parylene, c) Drilling of $500 \mu \mathrm{m}$ access holes, d) Dissolution of PMMA with acetone.

Final diameter of the fiber was found to increase with both increasing polymer molecular weight $(\mathrm{P}<0.025)$ and increasing polymer concentration $(\mathrm{P}<0.001)$, thus providing a mechanism to control fiber diameter (Fig. 5). The large error bars in Figure 5 indicate an unwanted variance in fiber diameter believed to be caused by PMMA buildup on the stylus when several fibers are drawn in rapid succession. To alleviate this unwanted fouling of the tip, alternative stylus materials were investigated including glass, Parylene®-coated tungsten, and perfluorooctyltrichlorosilane-coated (a nonstick material) tungsten. Table 1 summarizes this analysis, which demonstrated that a Parylene ${ }^{\circledR}-$ coated stylus yielded the best overall reduction in fiber diameter variation as evidenced by the lower standard deviation.

The direct-write method was also used to successfully draw fibers, notably in complex geometries including square "spirals," crisscrosses, and grids (Fig. 6) The primary advantage of this method is the elimination of the manual deposition of the pools at the terminal ends of each fiber (See Fig. 2). Eliminating this lowprecision step increased the accuracy of fiber terminal placement as well as expedited the entire fiber fabrication process through further automation. The direct-write method also eliminated the uncontrollable stylus buildup, which was suspected of contributing to the undesired variance in fiber diameter. With the direct-write method, the maximum fiber diameter standard deviation observed was $2.70 \mu \mathrm{m}$ with the $23 \% 495 \mathrm{k} \mathrm{g} / \mathrm{mol}$ solution, a value comparable to those obtained with the Parylene $®$-coated stylus.

Freely-suspended microchannels, as shown in figure 7, with I.D. ranging from $4 \mu \mathrm{m}$ to $100 \mu \mathrm{m}$ were fabricated via the procedure outlined in Figure 3. Electroosmotic flow was induced by loading the channels with buffer solution and applying a potential across the gold electrodes positioned at each end of the channel (Fig. 8). The velocity of the solution was observed to increase as the magnitude of the voltage was increased from $0 \mathrm{~V}$ to $1 \mathrm{kV}$. The direction of the flow reversed as the polarity of the electric field was switched, with the buffer solution moving toward the negative electrode. 


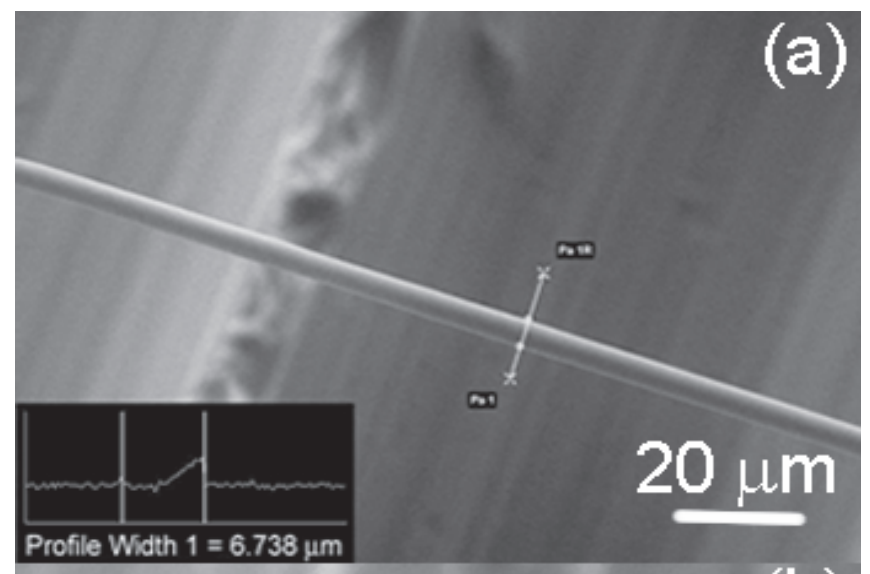

(b)
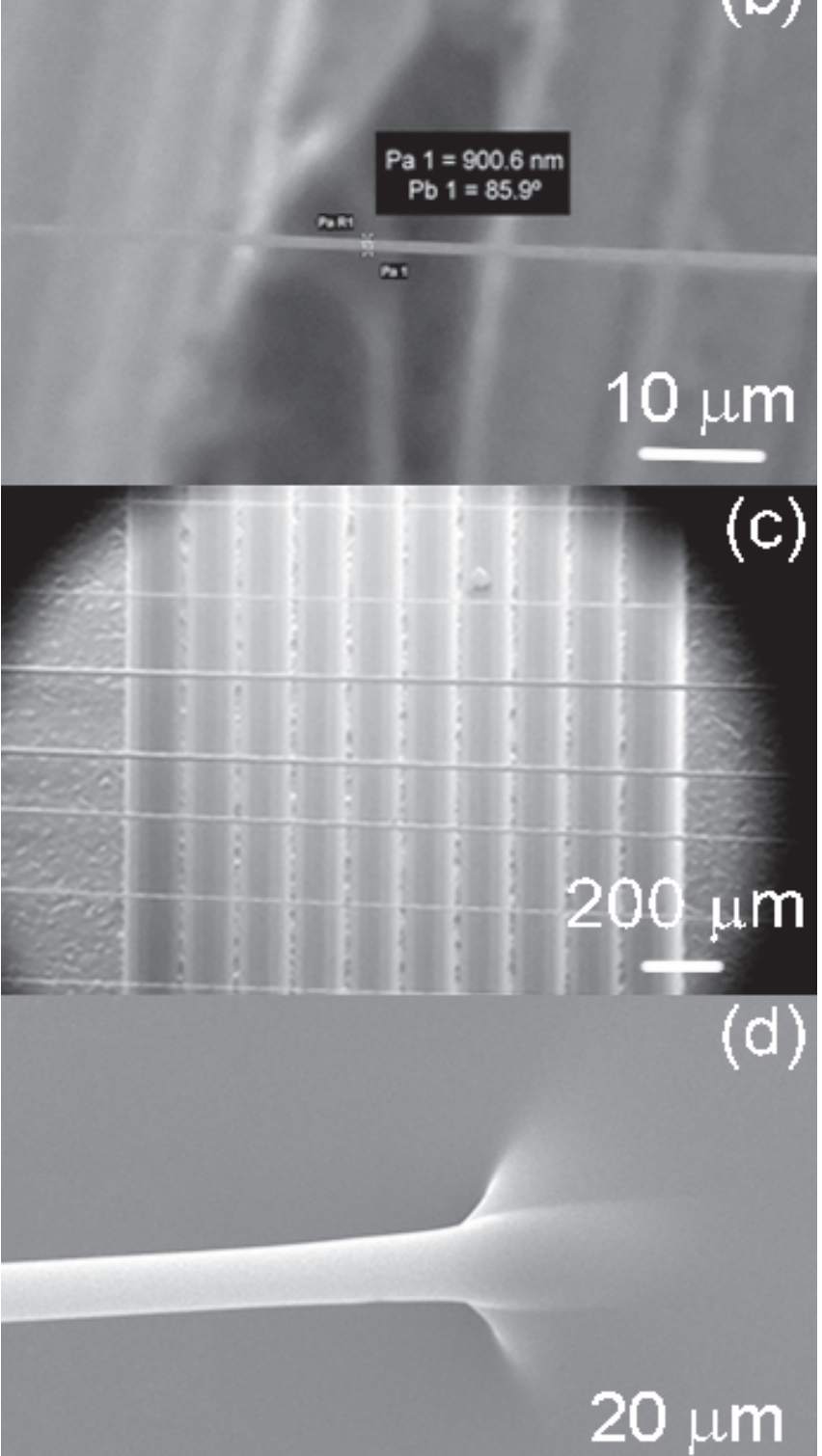

Figure 4. SEM images of polymer fibers drawn over $1.8 \mathrm{~mm}$ trench. (a) Suspended fiber drawn from $15 \%$ solution of $950 k$ $\mathrm{g} / \mathrm{mol}$ PMMA. (b) Suspended fiber drawn from 13\% solution of 950k g/mol PMMA. (c) Several parallel fibers drawn from $15.5 \%$ solution of $495 \mathrm{~kg} / \mathrm{mol}$ PMMA. (d) Tapered end of PMMA fiber.

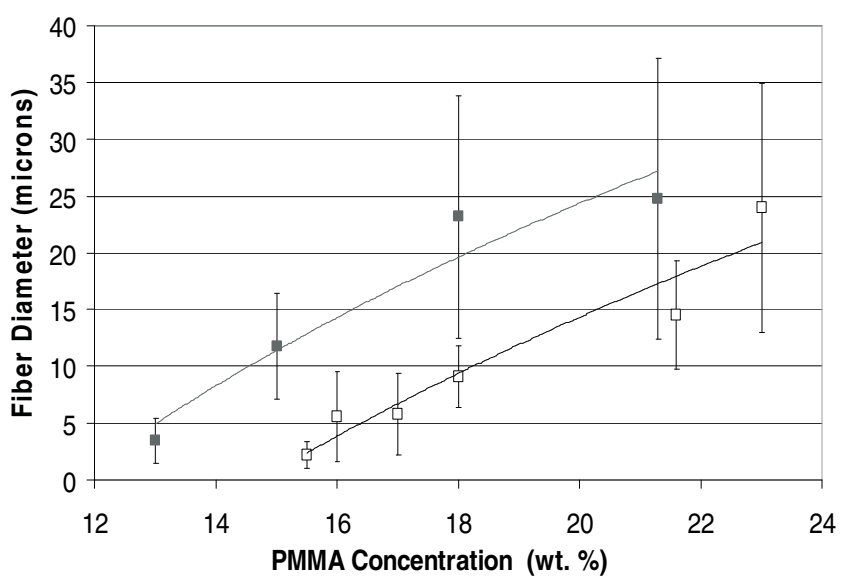

Figure 5. Fiber diameter versus concentration. The hollow and solid data points represent the average measured diameters of the drawn fibers for the $495 \mathrm{~kg} / \mathrm{mol}$ data and the $950 \mathrm{~g} / \mathrm{mol}$ data, respectively. The error bars represent the standard deviation of each data point.

\begin{tabular}{|c|c|c|c|c|}
\hline $\begin{array}{c}\text { Conc. } \\
(\text { wt.\% })\end{array}$ & $\begin{array}{c}\text { Tungsten } \\
(\mu \mathrm{m})\end{array}$ & $\begin{array}{c}\text { Glass } \\
(\mu \mathrm{m})\end{array}$ & $\begin{array}{c}\text { Parylene } \\
(\mu \mathrm{m})\end{array}$ & $\begin{array}{c}\text { Nonstick } \\
(\mu \mathrm{m})\end{array}$ \\
\hline 15.5. & $2.22 \pm$ & $2.43 \pm$ & No Data & $10.81 \pm$ \\
& 1.20 & 1.09 & & 6.88 \\
\hline 16 & $5.54 \pm$ & $4.38 \pm$ & $7.41 \pm$ & $8.76 \pm$ \\
& 3.95 & 3.11 & 2.49 & 2.84 \\
\hline 17 & $5.80 \pm$ & $4.20 \pm$ & $6.78 \pm$ & $7.92 \pm$ \\
& 3.59 & 2.04 & 1.38 & 5.08 \\
\hline 18 & $9.07 \pm$ & $4.84 \pm$ & $6.27 \pm$ & $4.69 \pm$ \\
& 2.68 & 2.71 & 3.02 & 2.69 \\
\hline 21.6 & $14.53 \pm$ & $18.37 \pm$ & $19.03 \pm$ & $15.24 \pm$ \\
& 4.82 & 6.36 & 1.40 & 7.58 \\
\hline 23 & $23.96 \pm$ & $17.29 \pm$ & $26.98 \pm$ & $34.80 \pm$ \\
& 11.00 & 5.43 & 2.03 & 11.61 \\
\hline
\end{tabular}

Table 1. Fiber diameter means and standard deviations for different stylus materials.

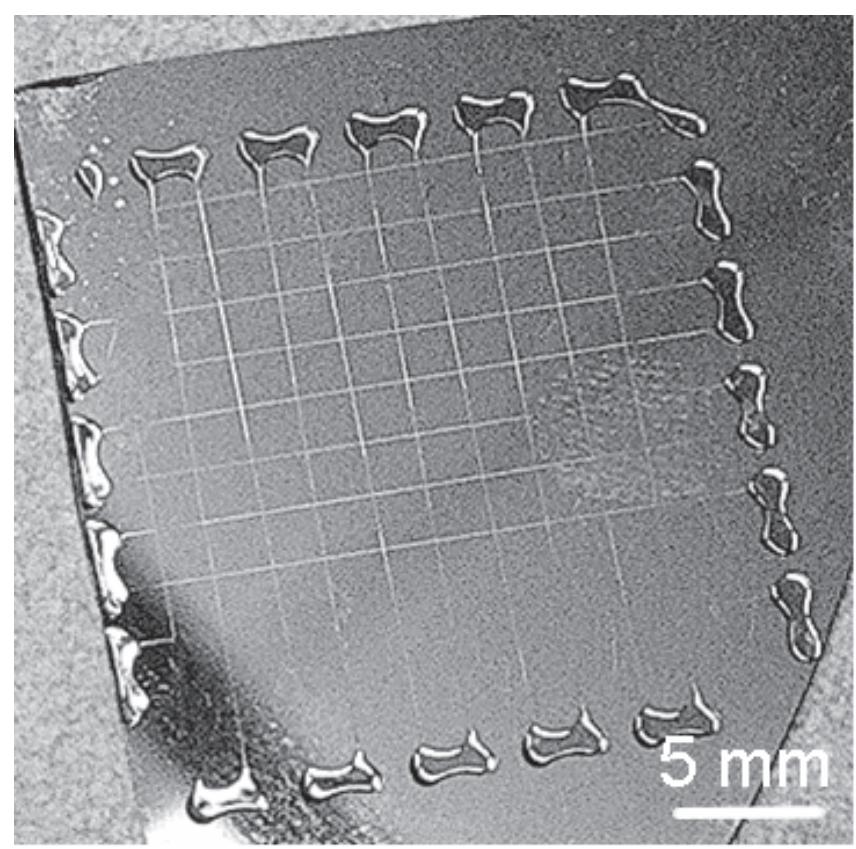

Figure 6. Pattern generated with direct-write method. 


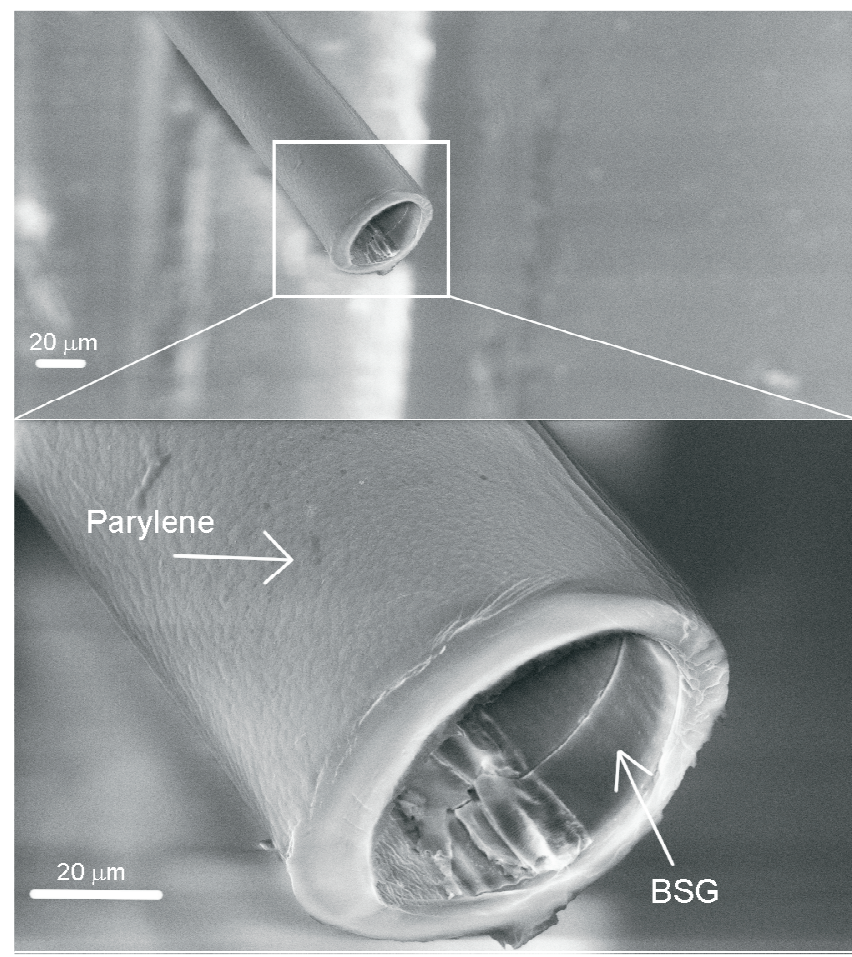

Figure 7. SEM images of cleaved microchannel.

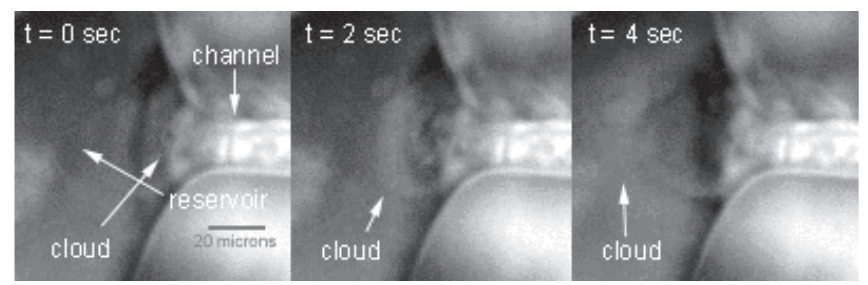

Figure 8. Ejection of fluorescent particle "cloud" from $20 \mu \mathrm{m}$ diameter electroosmotic flow channel.

\section{CONCLUSION}

Polymer micro- and nano- fibers can be drawn from a polymer solution using a stylus controlled by a custom-made ultrahigh-precision micromill. PMMA fibers ranging from sub-micron diameters to 100 microns have been successfully fabricated over a $1.8 \mathrm{~mm}$ trench on a silicon substrate. Characterization of the process indicated that fiber diameter increased as the solid concentration of the polymer solution increased. Additionally, it was shown that increasing the molecular weight of the PMMA resulted in larger fiber diameters.

A large variance between fibers drawn under the same experimental conditions was noted. A suspected culprit of this variance was the solidification of the solution on the stylus which accumulated during the drawing process. Several different stylus materials were tested in hopes of reducing this variance, and it was discovered that the stylus material did influence the severity of this variance, with a Parylene $®$-coated stylus being influenced the least by accumulation. It was also demonstrated that the stylus could be entirely replaced with a pressurized glass capillary filled with polymer solution. This micromill-controlled capillary was used to direct-write complex fiber configurations with low fiber diameter variance and high yield.

Fibers created with both the stylus-draw and direct-write methods were utilized as sacrificial layers in the construction of hollow microchannels. The walls of these channels consist of a thin, inner layer of BSG to make the channels hydrophilic as well as a thicker layer of Parylene ${ }^{\circledR}$ to improve structural integrity. Unbroken, freely-suspended channels were fabricated between two electrodes, enabling electroosmotic flow to be performed, paving the way for the development of more complex microfluidic devices.

\section{ACKNOLEDGEMENT}

This work was supported by NSF grant ECS0506941, NASA Cooperative Agreement NCC5-571, NSF EPSCoR grant 6016955, DOE EPSCoR grant 46411101095, and the Commonwealth of Kentucky.

\section{REFERENCES}

[1] Harfenist, S. A., Cambron, S. D., Nelson, E. W., Berry, S. M., Isham, A. W., Crain, M. M., Walsh, K. M., Keynton, R. S., Cohn, R. W. Direct Drawing of Suspended Filamentary Micro- and Nanostructures from Liquid Polymers. Nanoletters Vol. 4 No. 10 (2004) 1931-1937.

[2] Nain, A. S., Amon, C., Sitti, M. Three-Dimensional Nanoscale Manipulation and Manufacturing using Proximal Probes: Controlled Pulling of Polymer Micro/Nanofibers. Proceedings of the IEEE International Conference on Mechatronics. 3-5 (2004) 224-230.

[3] Friedrich, C., Vasile, M. Development of the Micromilling Process for High Aspect Ratio Microstructures. Journal of Microelectromechanical Systems. Vol. 5 No. 1 (1996) 33-38. 